\title{
Utility of Precession Electron Diffraction Patterns with Varying Degrees of Non- parallel Illumination from the Same Nominal Sample Area
}

\author{
Peter Moeck ${ }^{1}$, Jack C. Straton ${ }^{1}$, Danny J. Edwards ${ }^{2}$, and Ines Häusler ${ }^{3,1}$ \\ 1. Nano-Crystallography Group, Department of Physics, Portland State University, Portland, OR 97207- \\ 0751, USA \\ 2. Reactor Materials and Mechanical Design Group, Pacific Northwest National Laboratory, Richland, WA \\ 99354, USA \\ ${ }^{3 .}$ Department of Physics, Humboldt University Berlin, Newtonstraße 15, 12489 Berlin, Germany
}

Diffraction of a primary electron beam that is precessing, i.e. Precession Electron Diffraction (PED), in a Transmission Electron Microscope (TEM) [1-8], has over the last twenty-odd years become "an invaluable tool, not only for the determination of unknown crystal structures but also in aiding the analysis of local microstructure" [8]. Seven years after its first demonstration [9], the "scanning version of PED" [4-6] rivals in popularity electron backscattering in a scanning electron microscope.

The precession axis of the primary electron beam coincides ideally with the optical axis of the TEM and the (sharp) apex of the resulting hollow electron cone intersects the sample at a singular point. This ideal is, however, for a variety of reasons not readily achievable in practice. When the spherical aberration of the objective lens of the microscope is not corrected for and the alignment is not perfect, more or less blunt apexes of electron cones (that may not be completely hollow and round) result in PED. These cones intersect the sample over some area (rather than in a single point) and define the spatial resolution of the PED technique. The cones have also been observed to "wander" (or "wiggle and dance" [7]) over some sample area $[3,4]$. Correction of spherical aberration of the (probe forming) objective lens pre-field may reduce this "wandering" to approximately $1 \mathrm{~nm}$ [7].

Utilizing the recently derived relation $t_{l o c} \approx \lambda / 2\left\langle\left(g_{0}(\alpha+\beta)\right)^{2}\right\rangle-\langle\alpha+\beta\rangle\left\langle g_{0}(\alpha+\beta)\right\rangle$, where $t_{l o c}$ is the local crystal sample thickness, $\lambda$ is the electron wavelength, $g_{0}$ is the magnitude of the reciprocal lattice vector that determines the radius of the zero-order Laue circle, $\alpha$ is the precession half angle $(5-60 \mathrm{mrad})$, and $\beta$ is the convergence angle $(0.5-5 \mathrm{mrad})[10]$, one can in principle assess the size of these sample areas. This is because the radii of the observed zero-order Laue circles in PED patterns must for a plane-parallel sample plate fall on an "equal thickness" curve in Figure 1. Observed deviations from this functional relationship are indications that the sample is not a plane parallel plate and that the apex of the electron cone is blunt. Utilizing wedge shaped samples (that often arise naturally by the crushing of inorganic crystals), one can then assess the illuminated sample area (as a function of the precession angle) after one has determined the wedge angle by some appropriate technique such as the spacing of two-beam extinction fringes. (The linear increase with convergence angle and the quadratic increase with precession angle $[1,7,8]$ of the size of a blunt electron cone apex intersection with the sample may need to be considered in this context.)

In ref. [10] we proposed to utilize this relation (and its generalizations to higher order Laue zones) for the determination of those reflections that can be utilized straightforwardly for structural fingerprinting from PED patterns in a TEM [2]. That idea is based on Boris Vainshtein's critical thickness $\left(t_{c r i}\right)$ criterion for quasi-kinematic diffraction: $\Omega / \lambda \cdot t_{c r i} \leq\left|F_{h k l}\right|$, where $\Omega$ is the volume of the crystal's unit cell and $\left|F_{h k l}\right|$ is the square root of a reflection's intensity (i.e. a kinematical structure factor amplitude) [11]. Large precession angles lead generally to instantaneous "few-beam" conditions [2] that are better approximations to exact two-beam conditions (for which Vainshtein's inequality has been derived originally). For candidate crystal 
structures, $\Omega$ and $\left|F_{h k l}\right|$ will be known so that one needs to make sure that conditions of $t_{l o c} \leq t_{c r i}$ are fulfilled for those reflections that one utilizes for structural fingerprinting from PED patterns in a TEM (with fixed $\lambda$ ).

In summary, observed Laue circle radii in PED patterns as function of the degree of non-parallel illumination can be utilized on the basis of our ideas for both determination of the local sample thicknesses and assessments of the illuminated sample areas. Quasi-kinematic structural fingerprinting of nanocrystals from selected PED spot intensities is also possible on the basis of our ideas. As the precession angle magnitude has so far been rarely exploited as an experimental degree of freedom and because locally determined sample thicknesses are quite valuable, it is safe to predict that these kinds of ideas will prove their worth in the future in a range of TEM investigations of crystalline materials (including 6D electron tomography [12], which covers direct and reciprocal space simultaneously) [13].

[1] R. Vincent and P. Midgley, Ultramicroscopy 53 (1994) 271.

[2] P. Moeck and S. Rouvimov, Zeitschrift für Kristallographie 225 (2010) 110, open access:

http://www.degruyter.com/view/j/zkri.2010.225.issue-2-3/zkri.2010.1162/zkri.2010.1162.xml

[3] Y. Liao and L. D. Marks, Ultramicroscopy 117 (2012) 1.

[4] P. Moeck et al, Proc. $11^{\text {th }}$ IEEE International Conference on Nanotechnology, August 15-18, 2011, Portland, Oregon, USA, pp. 754-759, DOI: 10.1109/NANO.2011.6144300

[5] P. Moeck et al, Cryst. Res. Technol. 46 (2011) 589.

[6] A. S. Eggeman and P. A. Midgley, Adv. Imaging Electron Phys. 170 (2012) 1.

[7] A. S. Eggeman et al, Zeitschrift für Kristallographie - Crystalline Materials 228 (2012) 43.

[8] P. Midgley and A. Eggeman, IUCrJ 2 (2015) 126; open access:

http://journals.iucr.org/m/issues/2015/01/00/ro5004/ro5004.pdf

[9] E. F. Rauch et al, Microsc. Anal. 22 (2008) S5.

[10] P. Moeck et al, Microsc. Microanal. 18 (Suppl. 2), 2012, p. 562.

[11] B. K. Vainshtein, Fundamentals of Crystallography, Symmetry and Methods for Structural

Crystallography (Springer, $2^{\text {nd }}$ edition, 1994) p. 345; see also B. K. Vainshtein, Acta Cryst. B 47 (1991) 145. [12] A. Eggeman et al, Acta Cryst. A 70 (2014) C368.

[13] The first author acknowledges discussions with Prof. Dr. rer. nat. habil Wolfgang Neumann and members of Wolfgang's former research group at the Department of Physics of Humboldt University, Berlin/Germany, where he worked on sabbaticals in 2010 and the summer of 2012.

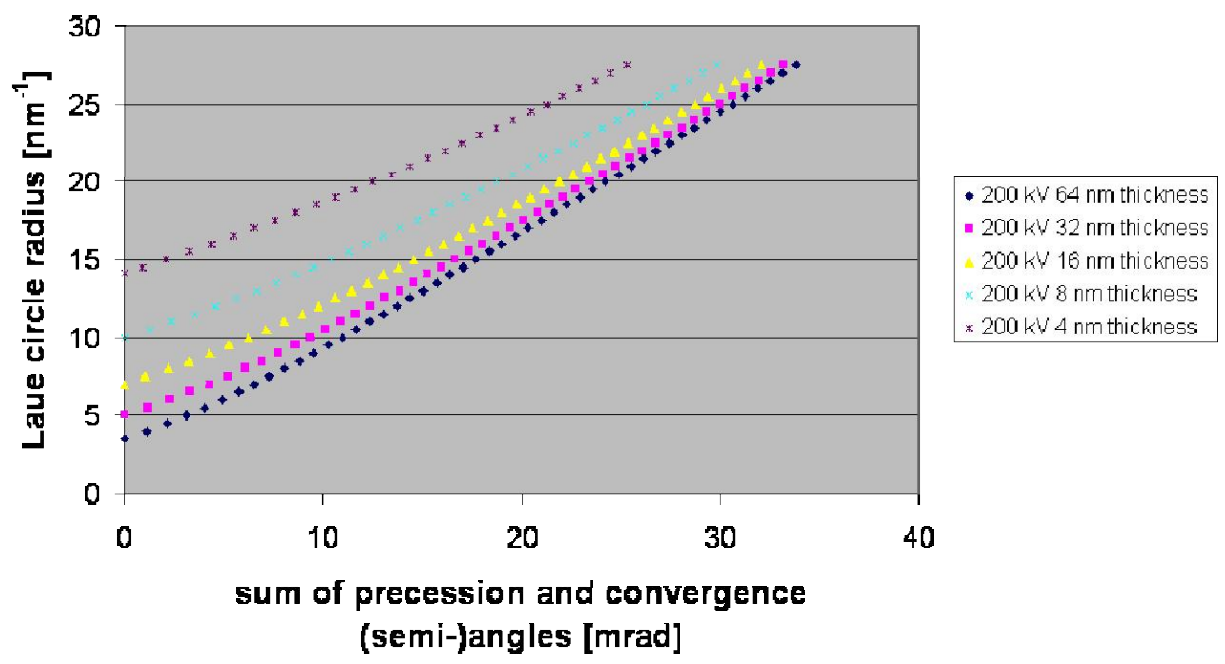

Figure 1. Predicted zero-order Laue circle radii in PED patterns versus degree of non-parallel illumination for plane parallel crystal plate samples with thicknesses between 4 and $64 \mathrm{~nm}$. 